\title{
Deep-time convergent evolution in animal communication presented by shared adaptations for coping with noise in lizards and other animals 10
}

\author{
Terry J. Ord ${ }^{1}$ (D) | Danielle A. Klomp ${ }^{1} \quad$ | Thomas C. Summers ${ }^{1} \quad$ Arvin Diesmos $^{2}$ | \\ Norhayati Ahmad $^{3}$ | Indraneil Das ${ }^{4}$
}

${ }^{1}$ Evolution \& Ecology Research Centre and the School of Biological, Earth and Environmental Sciences, University of New South Wales, Sydney, NSW, Australia

${ }^{2}$ Herpetology Section, Zoology Division, National Museum of the Philippines, Manila, Philippines

${ }^{3}$ Department of Biological Sciences and Biotechnology, Universiti Kebangsaan Malaysia, Bangi, Malaysia

${ }^{4}$ Institute of Biodiversity and Environmental Conservation, Universiti Malaysia Sarawak, Kota Samarahan, Malaysia

\section{Correspondence}

Terry J. Ord, Evolution \& Ecology

Research Centre and the School of

Biological, Earth and Environmental

Sciences, University of New South Wales,

Sydney, NSW, Australia.

Email: t.ord@unsw.edu.au

Funding information

University of New South Wales (UNSW)

Editor: Greg Grether

\begin{abstract}
Convergence in communication appears rare compared with other forms of adaptation. This is puzzling, given communication is acutely dependent on the environment and expected to converge in form when animals communicate in similar habitats. We uncover deep-time convergence in territorial communication between two groups of tropical lizards separated by over 140 million years of evolution: the Southeast Asian Draco and Caribbean Anolis. These groups have repeatedly converged in multiple aspects of display along common environmental gradients. Robot playbacks to free-ranging lizards confirmed that the most prominent convergence in display is adaptive, as it improves signal detection. We then provide evidence from a sample of the literature to further show that convergent adaptation among highly divergent animal groups is almost certainly widespread in nature. Signal evolution is therefore curbed towards the same set of adaptive solutions, especially when animals are challenged with the problem of communicating effectively in noisy environments.
\end{abstract}

\section{K E Y W O R D S}

adaptive differentiation, contextual plasticity, historical contingency, repeated evolution, robotic playbacks

\section{INTRODUCTION}

Animals relying on signals for similar functions-for example, attracting mates or defending territories - and trying to communicate under similar environmental conditions are expected to converge in signal characteristics (Marler, 1967). This is because natural selection should only favour those strategies that maximise the detection and recognition of communicative signals in those environments (Endler, 1992). For example, communication theory (Dusenbery, 1992; Wiley, 2006) posits that animals should produce signals that maximise the signal-to-noise ratio with the environment through changes in the intensity of signalling or redundancy of information conveyed. Yet the convergent evolution of common communicative strategies does not appear to be as likely as convergence in morphology (Ord \& Summers, 2015; York \& Fernald, 2017; these two studies examined over 120 examples of convergent, parallel and functionally redundant adaptations repeatedly arising from independent evolution). This is despite behaviour putatively being more evolutionarily labile than morphology (Blomberg et al., 2003) and efficient communication being central to the reproductive fitness of many animals (Bradbury \& Vehrencamp, 2011). However, this could be because there are countless ways in which animals might maximise signal-to-noise ratios, making signal convergence improbable.

Of the examples of convergence in animal communication, most are between populations of the same species 